\title{
Comportamento dos nervos glossofaríngeo e vago, na região retrofaríngea de ovinos: origem aparente no crânio, trajeto, ramificação e distribuição ${ }^{1}$
}

\author{
Márcia Maria M.D. Farias ${ }^{2}$, Cristiane V. Wenceslau², Juliana Passos ${ }^{2}$, Dulcinéa G. \\ Teixeira $^{2}$, Karla P.C. Araújoº ${ }^{2}$, Carlos E. Ambrósio ${ }^{2 *}$, Daniele S. Martins ${ }^{2}$, Maria \\ Angélica Miglino ${ }^{2}$ e Irvênia L.S. Prada ${ }^{2}$
}

\begin{abstract}
Farias M.M.M.D, Wenceslau C.V., Passos J., Teixeira D.G., Araújo K.P.C., Ambrósio C.E., Martins D.S. \& Prada I.L.S. 2007. [Behavior of the glossopharyngeal and vagus nerves in the retropharyngeal region of sheep: apparent origin in cranium, course, branching and distribution.] Comportamento dos nervos glossofaríngeo e vago, na região retrofaríngea de ovinos: origem aparente no crânio, trajeto, ramificação e distribuição. Pesquisa Veterinária Brasileira 27(3):115-123. Setor de Anatomia dos Animais Domésticos e Silvestres, Departamento de Cirurgia, Faculdade de Medicina Veterinária e Zootecnia. Universidade de São Paulo, Avenida Prof. Dr. Orlando Marques de Paiva 87, Cidade Universitária, São Paulo, SP 05508-900, Brazil. E-mail: ceambrosio@usp.br

In 60 hemiheads of sheep of the Santa Ines breed the apparent origin in the skull of itinerary, ramification and distribution of the glossopharingeal nerve (Gf) and the vagus nerve $(\mathrm{Vg})$ in the retropharyngeal region $(\mathrm{Rr})$ were studied. By fixation and dissection of the specimens it was seen that the glossopharyngeal nerve and the vagus nerve arise from the jugular foramen in $100 \%$ of the cases. The right and the left glossopharingeal nerve (Glde) are frequently $(86.6 \%)$ located more medially to the tympanic bulla, pass caudally to the stylohyoid bone, bypass the margin of the caudal stylopharyngeal muscle, the tonsilla, of the pharyngeal and the lingual mucous membrane. These branches are distributed, respectively, in the carotid sinus, pharyngeal musculature, soft palate, stylopharyngeal muscle, palatine tonsil, pharyngeal mucosa and the caudal third of the tongue (100\%). The right and the left vagus nerve run caudally in association with the accessory nerves (Ac) up to the atlas (70\%) and go then medially to the occipital artery, dorsally to the common carotid and the sympathetic trunk in the cervical region $(80 \%)$. The vague nerves have pharyngeal $(86.6 \%)$ and cranial laryngeal $(100 \%)$ branches.
\end{abstract}

INDEX TERMS: Anatomy, sheep, glossopharingeal nerve, vagus nerve.

RESUMO.- Em 60 hemicabeças de ovinos da raça Santa Inês foram estudadas a origem aparente no crânio, trajeto, ramificação e distribuição do nervo glossofaríngeo (Gf) e do nervo vago $(\mathrm{Vg})$, na região retrofaríngea $(\mathrm{Rr})$. Mediante fixação e dis-

\footnotetext{
${ }^{1}$ Recebido em 17 de outubro de 2006.

Aceito para publicação em 12 de fevereiro de 2007.

Parte da Tese de Doutorado do primeiro autor, apresentado ao Curso de Pós-Graduação em Anatomia dos Animais Domésticos e Silvestres, Faculdade de Medicina Veterinária e Zootecnia (FMVZ), Universidade de São Paulo (USP).

2 Setor de Anatomia dos Animais Domésticos e Silvestres, Departamento de Cirurgia, FMVZ, USP, Av. Prof. Dr. Orlando Marques de Paiva 87, Cidade Universitária, São Paulo, SP 05508-900, Brasil. *Autor para correspondência: ceambrosio@usp.br
}

secação das peças, foi observado que os nervos glossofaríngeos e vagos originam-se no forame jugular em $100 \%$ dos casos. Os nervos glossofaríngeos direito e esquerdo (Gfde) são localizados, com maior frequiência $(86,6 \%)$, medialmente à bula timpânica, passam caudalmente ao osso estiloióide, contornam a margem caudal do músculo estilofaríngeo caudal, tonsilar, da mucosa faríngea e lingual. Estes ramos distribuemse, respectivamente, no seio carotídeo, musculatura faríngea, palato mole, músculo estilofaríngeo caudal, tonsila palatina, mucosa faríngea e terço caudal da língua (100\%). Os nervos vagos direito e esquerdo (Vgde) correm caudalmente em associação com os nervos acessórios (Ac) até o atlas (70\%), após o que passam medialmente à artéria occipital, dorsalmente à carótida comum e ao tronco simpático, na região cervical $(80 \%)$. Os ra- 
mos dos nervos vagos são os faríngeos $(86,66 \%)$ e os laríngeos craniais $(100 \%)$.

TERMOS INDEXADOS: Anatomia, ovinos, nervo glossofaríngeo, nervo vago.

\section{INTRODUÇÃO}

A topografia e a anatomia descritiva dos nervos glossofaríngeo (Gf) e vago (Vg) é de grande interesse, e existe uma complexidade anatômica considerável em sua distribuição e ramificação. A descrição anatômica destes nervos e da região retrofaríngea ( $\mathrm{Rr}$ ), contribuirá nos casos clínicos e cirúrgicos, considerando-se a possibilidade de ocorrência de diferenças anatômicas entre as espécies e mesmo entre indivíduos de mesma espécie.

De acordo com Moore (1990) o espaço retrofaríngeo é virtual envolvido na atuação da faringe, laringe, traquéia e esôfago durante a deglutição. Está localizado na parte posterior à faringe, e é limitado anteriormente pela fáscia bucofaríngea, na parte posterior pela lâmina pré-vertebral e na parte lateral pela bainha carótida, estendendo-se da base do crânio até o mediastino superior. Por meio do espaço retrofaríngeo, também denominado região retrofaríngea $(\mathrm{Rr})$ pelos autores Godinho (1973 a, b), Davidson et al. (1981) e Stocker et al. (1997), até o pescoço e a língua, chegam quatro nervos cranianos: IXglossofaríngeo, X- vago, XI- acessório e o XII- hipoglosso, os quais são as vias motoras e sensitivas principais dos músculos e mucosa da faringe, laringe e língua. Outras estruturas evidenciadas na região retrofaríngea são: artéria occipital, artéria carótida interna e glânglio cervical cranial que integram a via simpática dos componentes da cabeça emitindo os nervos carotídeos interno e externo.

Em ovinos os nervos glossofaríngeo e vago emergem da cavidade craniana através do forame jugular (Dougherty et al. 1958, Habel 1970, Getty, 1975, Godinho et al. 1987, Dyce et al. 1990). Dougherty et al. (1958) descrevem que o Gf após emergir, da região caudoventral com extensão de $1 \mathrm{~cm}$ até a bula timpânica, emite o ramo do seio carotídeo. $\mathrm{O} \mathrm{Vg}$, antes de se separar do Ac emite uma anastomose para o Gf, corre no sentido caudal e recebe uma grande anastomose do nervo laríngeo cranial.

Dada a complexidade anatômica e a importância funcional da $\mathrm{Rr}$ e das estruturas nela contidas, comparado com a escassez de literatura referente ao tema, despertou interesse em descrever estruturalmente e topograficamente a Rr. Desta forma, o presente trabalho visa estabelecer a delimitação da $\mathrm{Rr}$ e a identificação dos nervos glossofaríngeo e vago, e seus respectivos ramos, assim como conhecer a disposição anatômica mais freqüente. Como resultado serão obtidos subsídios anatômicos que permitirão escolher a melhor via de acesso à $\mathrm{Rr}$ nos procedimentos clínicos e cirúrgicos nestes animais para colaborar com a literatura nacional.

\section{MATERIAL E MÉTODOS}

\section{Microscopia}

Nesta pesquisa foram utilizados 30 ovinos (Ovis áries, Linnaeus 1758) da raça Santa Inês, 9 fêmeas e 21 machos, de diferentes idades, procedentes de criações do Município de Ipirá, Bahia, coletados em frigoríficos do mesmo estado.

Após o abate, a cabeça e o pescoço foram isolados, e levados para o laboratório de Anatomia do Departamento de Anatomia dos Animais Domésticos da Escola de Medicina Veterinária da Universidade Federal da Bahia. Em seguida as peças foram fixadas e canuladas, através da artéria carótida comum com solução de formaldeído a $10 \%$. As estruturas vasculares foram injetadas, com látex Globo Tintas ${ }^{\circledR}$-Guarulhos e coradas com pigmento vermelho específico, para as artérias carótidas comuns, e azul para a veia jugular externa.

Para melhor visualização da Rr e comportamento do Gl e Vg foi realizado o corte sagital mediano preservando sua antimeria.

A delimitação da $\mathrm{Rr}$ foi feita mediante dissecação, para posterior descrição das estruturas nela contida. $O$ espaço virtual onde localiza esta região é delimitado: dorsalmente, a fáscia profunda que reveste a musculatura da faringe, os músculos laríngeos extrínsecos e a porção cranial do esôfago; ventral aos músculos longo e reto ventral da cabeça; caudalmente, ao plano transversal que tangencia a extremidade rostral da epiglote; rostromedialmente, quanto ao linfonodo retrofaríngeo medial e na posição lateral à glândula mandibular.

A dissecação das peças foi feita com auxílio de lupa bi-focal LP2311 (1,8X - 2,3X) evidenciando o percurso dos nervos, sob forma de desenhos esquemáticos e fotodocumentação. Para facilitar a visualização, os segmentos nervosos foram destacados com corante amarelo para o Gf e laranja para o Vg.

\section{Microscopia de Luz}

Em três hemicabeças foram coletados fragmentos dos nervos e da parede da faringe. Os mesmos foram processados utilizando-se a técnica histológica clássica para inclusão em parafina, cortes seriados em micrótomo (American Optical ${ }^{\circledR}$, modelo 820) com $5 \mu \mathrm{m}$ de espessura e corados com hematoxilina-eosina. 0 equipamento usado foi um microscópio binocular de luz, marca Zeiss, modelo Standard, acoplado com fotomicroscópio, marca Zeiss, modelo Axioskop, para registrar as amostras histológicas em fotografia.

\section{RESULTADOS}

Origem aparente dos nervos vago e glossofaríngeo no crânio

Em todas as preparações $(100, \%)$ os nervos glossofaríngeo e vago se originam da superfície ventrolateral do bulbo, abaixo da margem ventral do pedúnculo cerebelar caudal, perfurando a dura-máter e emergindo do crânio através do forame jugular ao longo de sua margem caudal (Fig.1 e 2).

\section{Trajeto dos nervos glossofaríngeos direito e esquerdo}

Em $100 \%$ dos casos, o antímero direito (AD) e o antímero esquerdo $(\mathrm{AE})$ se postam medialmente à bula timpânica e lateralmente ao nervo carotídeo interno. O AD, em $43,33 \%$ das oportunidades, e o $\mathrm{AE}$ em $50 \%$, contornam a margem caudal do músculo estilofaríngeo caudal seguindo no sentido rostral, entre o osso estiloióideo e o músculo estilofaríngeo caudal; perfuram o músculo hiofaríngeo, atingindo a faringe para alcançar a base da língua. O AD (5\%), é acompanhado pelo Vg e Ac. Em $28,3 \%$ no $A D$ e $26,6 \%$ no $A E$ foi encontrado o gânglio laterofaríngeo ao passar pela parede faríngea por meio do músculo hiofaríngeo, dorsal à origem do ramo tonsilar (Fig.1 e 2). 
Fig.1. Hemicabeça de ovino: comportamento dos nervos glossofaríngeo e vago. (A) corte sagital mediano, vista face esquerda: nervo glossofaríngeo (Gf) e vago (Vg); Cc artéria carótida comum; GCC- gânglio cervical cranial: Gd- gânglio distal do nervo vago; Gd- gânglio distal. (B) vista face lateral direita, o Vg emite o nervo laríngeo cranial (NLC), do qual se destaca o ramo externo (rE), segue em direção caudal na artéria laríngea cranial e fissura tireóidea (ft); Tf- músculo tireofaríngeo; Cf- músculo circofaríngeo; Ti- músculo tiroiódeo. (C) corte sagital mediano vista face direita, os nervos Gf e Vg surgem das radículas; os nervos Gf dividem-se em ramos do seio carotídeo (rSC), faríngeo (rF) e do músculo estilo-faríngeo caudal (rEfC), como ramos colaterais e os ramos tonsilar (rT) da mucosa (rM) e lingual (rL).

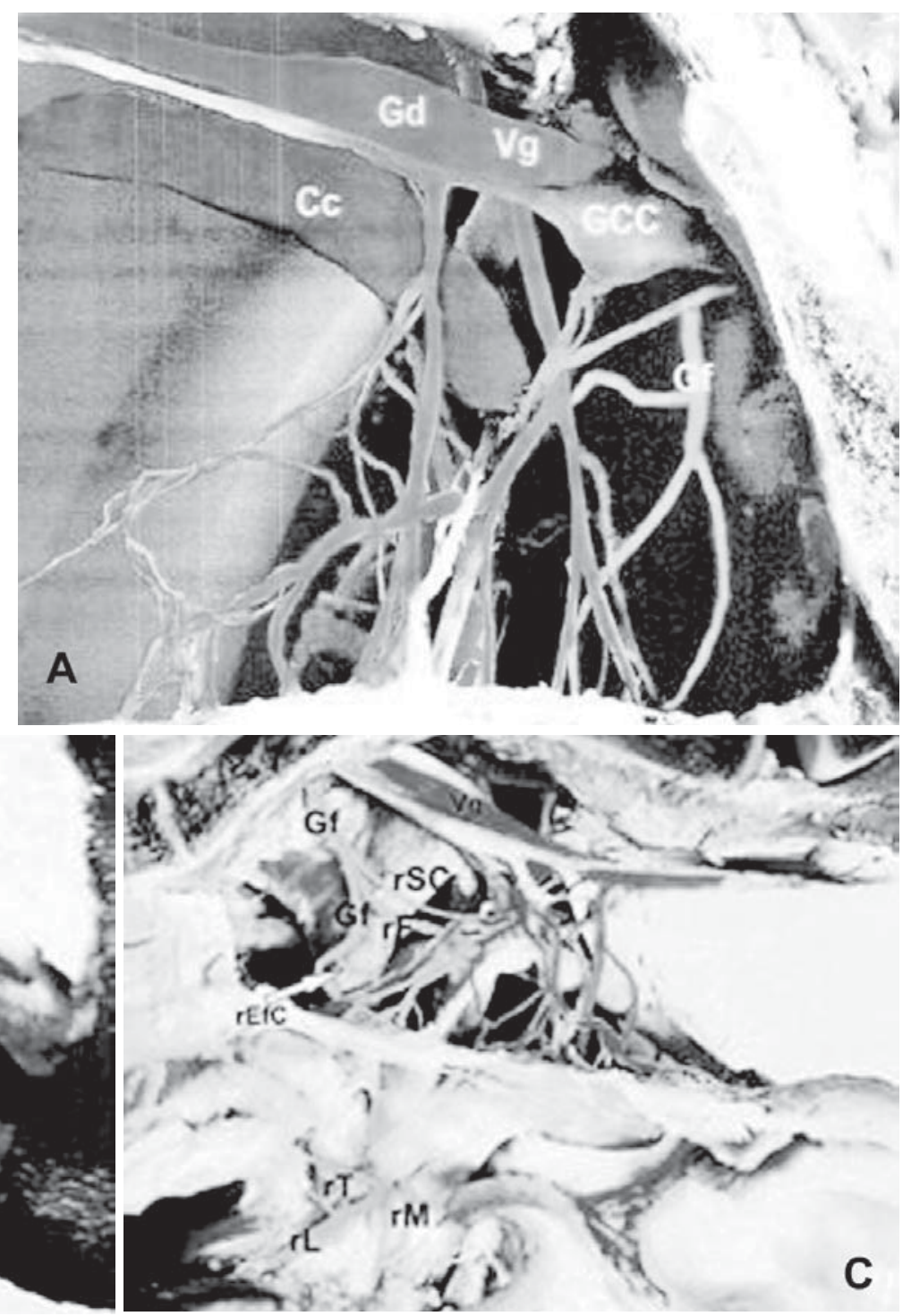

\section{Origem individualizada dos ramos colaterais dos nervos glossofaríngeos}

Em 53,3\% das amostras os ramos dos seios carotídeos foram identificados como os primeiros ramos colaterais dos Gfde. Estes ramos destacam-se em 46,6\% dos casos do Gf correspondendo à emergência dos ramos faríngeos direito $\mathrm{e}$ esquerdo. Os ramos do seio carotídeo originam-se em 43,3\% das oportunidades correspondendo à face medial do músculo occipitohióideo (Fig.1 e 2).

Os ramos faríngeos foram identificados como a segunda ramificação dos Gfde e o $\mathrm{AD}$, em $46,6 \%$ dos casos, e o $\mathrm{AE}$, em $43,3 \%$, surgem dos Gfde, na face medial do músculo occipitohióideo (Fig.1 e 2).

Os ramos do músculo estilofaríngeo caudal representam a terceira ramificação dos Gfde. Em 56,6\% das amostras o $\mathrm{AD}$ e o $\mathrm{AE}$, originam-se do terço médio da margem caudal do músculo estilofaríngeo caudal. Estes ramos surgem a partir do terço distal da margem caudal do músculo estilofaríngeo, para ambos os antímeros (25\%) (Fig.1 e 2).

\section{Trajeto dos ramos colaterais dos nervos glossofaríngeos direito e esquerdo}

No $\mathrm{AD}(25 \%)$ e no $\mathrm{AE}(26,6 \%)$ foi observado que os ramos do seio carotídeo surgem isoladamente ao nível da face medial do músculo occipitoióideo. Percorrem a direção caudal, medialmente ao gânglio cervical cranial aos ramos faríngeos e faringoesofágicos dos Vg e terminam na face rostrolateral das artérias occipitais. Enquanto em 38,3\% em ambos os antímeros os ramos do seio carotídeo destacam-se ao mesmo tempo com os ramos faríngeos e apresentam o mesmo trajeto e distribuição (Fig.1 e 2).

Os ramos faríngeos apresentam-se isolados ou em conjunto com os ramos do seio carotídeo, seguem no sentido rostroventral na face medial do músculo occipitohióideo, cruzam as artérias carótidas externas rostralmente aos nervos glossofaríngeos (Gf) e distribuem-se nos músculos da faringe e palato mole, em $25 \%$ e $21,6 \%$ dos casos referentes, respectivamente, ao $\mathrm{AD}$ e $\mathrm{AE}$. No $\mathrm{AD}(25 \%)$ e no $\mathrm{AE}$ $(26,6 \%)$, contudo, seguem sempre caudalmente aos Gf(Fig.1 e 2).

Os ramos dos músculos estilofaríngeos caudais em no $\mathrm{AD}$ $28,3 \%$ e em $30 \%$ no AE dirigem-se no sentido rostroventral atingin- 


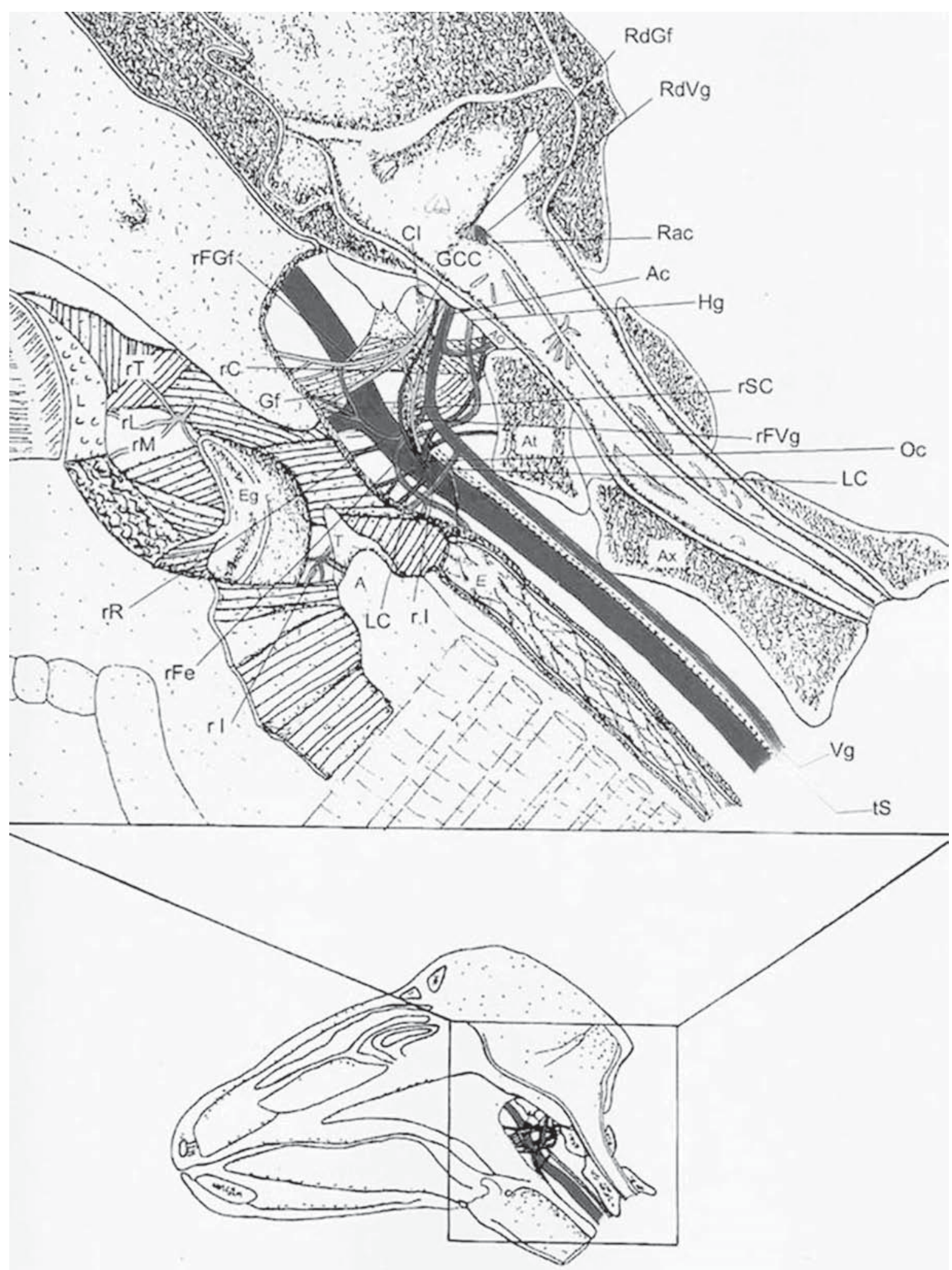

Fig.2. Esquema representativo da origem aparente no crânio, trajeto, ramificação e distribuição dos nervos glossofaríngeo (Gf) e vago (Vg) de ovinos da raça Santa Inês. Observa-se as seguintes estruturas: cartilagem aritenóide (A), nervo acessório (Ac), atlas (At), áxis (Ax), nervo carótido interno (Cl), epiglote (E), glânglio cervical cranial (GCC), nervo hipoglosso (Hg), artéria laríngeo cranial (LC), artéria occipital (Oc), ramo comunicante $(\mathrm{rC})$, ramo interno $(\mathrm{rl})$, ramo lingual $(\mathrm{rL})$, ramo da mucosa faríngea $(\mathrm{rM})$, ramo rostral do ramo faringoesofágico $(\mathrm{rR})$, ramo tonsilar $(\mathrm{rT})$, raiz do acessório (Rac), ramo faringoesofágico $(\mathrm{rFe})$, ramo do seio carotídeo ( $\mathrm{rSC}$ ), radículas do glossofaríngeo (RdGf), radículas do vago $(\mathrm{rdVg})$, ramo faríngeo do glossofaríngeo ( $\mathrm{rFGf}$, ramo faríngeo do vago $(\mathrm{rFVg})$, cartilagem tireóide $(\mathrm{T})$, tronco simpático $(\mathrm{tS})$. 


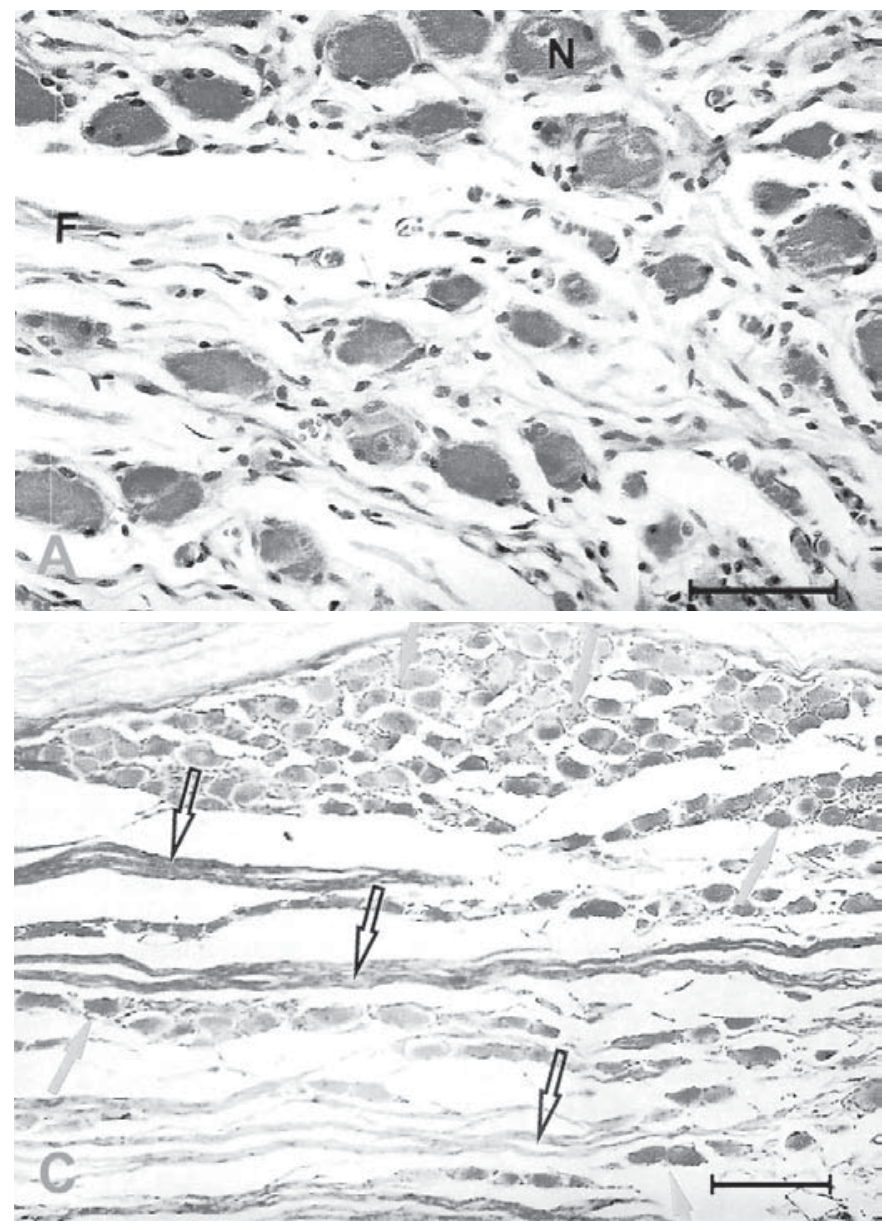

Fig.3. Aspecto histológico de corte longitudinal dos segmentos. Coloração Hematoxilina-Eosina. (A) Nervo glossofaríngeo, ao nível da origem do ramo tonsilar, evidenciando os neurônios $(\mathrm{N})$ distribuídos entre feixes de fibras nervosas $(\mathrm{F})$; barra: $16 \mu \mathrm{m}$. (B) Nervo glossofaríngeo, ao nível da origem do ramo tonsilar, observando-se $\mathrm{N}$ envoltos por células satélites (setas cheias); notar núcleos e (seta vazia) e nucléolos (seta pequena); barra: $16 \mu \mathrm{m}$. (C) Nervo vago, observando-se agregados de corpos de neurônios (setas cheias), indicativos de formação ganglionar, entrecruzados por feixes nervosos (setas vazias); barra: $50 \mu \mathrm{m}$. (D) Nervo vago, registrando agregado de $\mathrm{N}$ com seus núcleos (seta grande) e nucléolos (seta pequena), além de $\mathrm{F}$ dispostas longitudinalmente; barra: $16 \mu \mathrm{m}$. (E) Fragmento de parede dorsal da faringe, evidenciando-se estruturas glandulares com células serosas (seta cheia) e mucosas (seta vazia), além de fibras musculares estriadas (E) e tecido adiposo (A); barra: $50 \mu \mathrm{m}$.

do os músculos estilofaríngeos caudais no terço médio da margem caudal. Em $10 \%$ dos casos do AD e $15 \%$ do AE, os ramos dos músculos estilofaríngeos caudais distribuem-se no terço distal da margem caudal dos músculos estilofaríngeos caudais (Fig.1 e 2).

\section{Distribuição dos ramos retrofaríngeos dos nervos glosso- faríngeos direito e esquerdo: ramos do seio carotídeo, ra- mos faríngeos e ramos do músculo estilofaríngeo caudal}

Verificou-se que em $100 \%$ das amostras os ramos do seio carotídeo distribuem-se na face rostrolateral das artérias

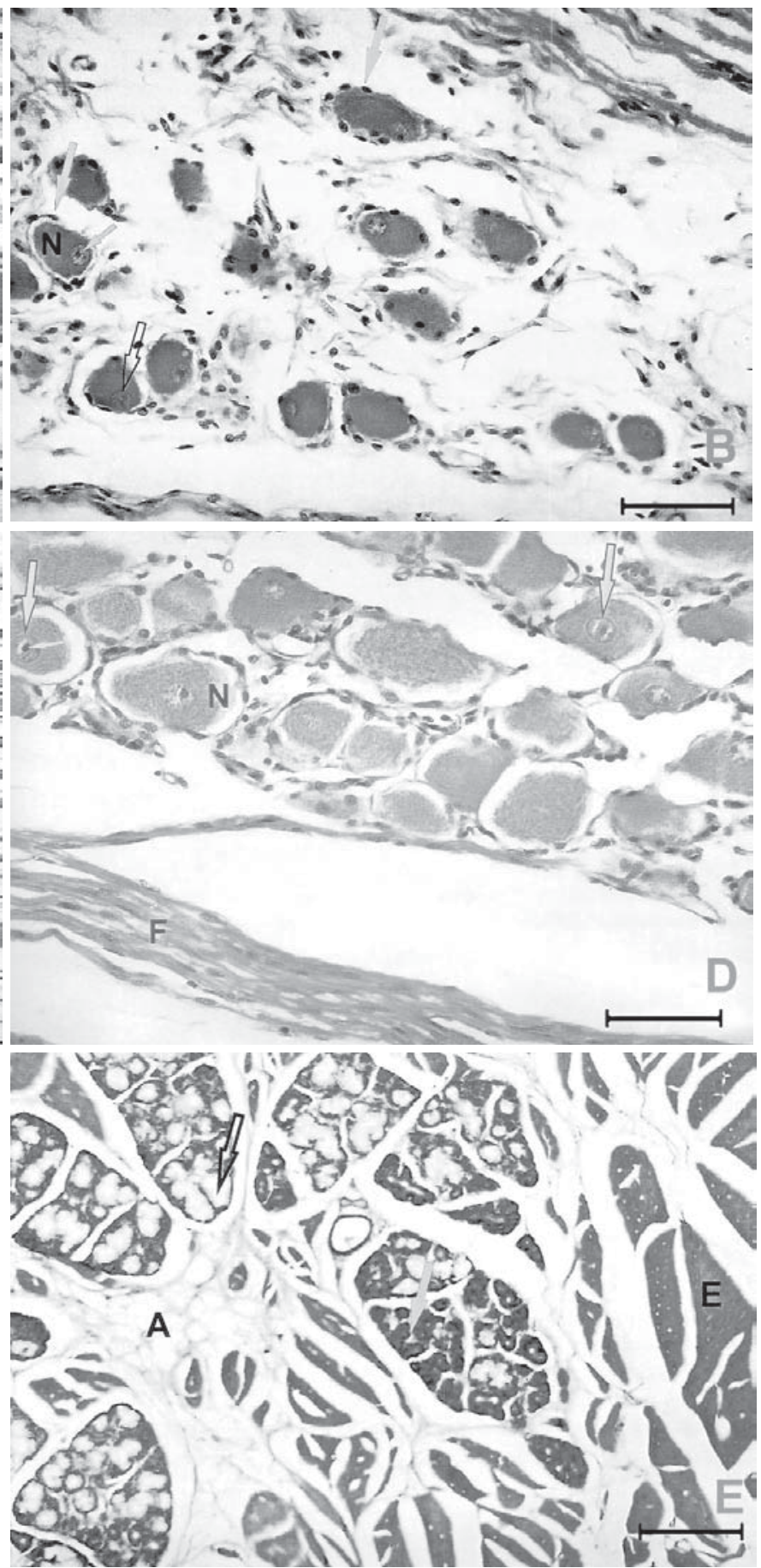

occipital e carótida interna, em correspondência à origem. Os ramos faríngeos distribuem-se nos músculos da faringe. Os ramos dos músculos estilofaríngeos caudais em $100 \%$ dos casos seguem até o músculo estilofaríngeo caudal (Fig.1 e 2).

Ramos terminais dos nervos glossofaríngeos direito e esquerdo: origem individualizada dos ramos terminais dos nervos glossofaríngeos direito e esquerdo

Os Gfde, em 93,3\% das amostras, após emitirem os ramos do seio carotídeo, ramos faríngeos e dos ramos dos múscu- 
los estilofaríngeos caudais atravessam o músculo hiofaríngeo, abandonando-o e terminando em ramos tonsilares. Os ramos da mucosa, em todas as peças, destacam-se dos Gfde, após sua passagem através do músculo hiofaríngeo, relacionam-se com a emergência dos ramos tonsilares, e seguem, em sentido caudoventral, para se distribuir na mucosa faríngea. Os ramos linguais destacam-se dos Gfde, imediatamente aos ramos tonsilares, em correspondência à base da língua, penetrando-a em sentido rostral (100\%) (Fig.1).

Trajeto e distribuição dos ramos terminais dos nervos glossofaríngeos direito e esquerdo: ramos tonsilares, ramos da mucosa, ramos linguais

Em $100 \%$ dos casos os ramos tonsilares se deslocam em direção da tonsila palatina, distribuindo-se por toda sua extensão. Da mesma forma, os ramos da mucosa ramificam-se na base da língua e nas paredes laterais da orofaringe. Os ramos linguais se originam após a emergência dos ramos tonsilares e distribuem-se no terço caudal da língua (Fig.1).

\section{Trajeto dos nervos vagos direito e esquerdo}

O Vago em ambos os antímeros surge da cavidade craniana, juntamente com o Gf e Ac, visto que o Vg corre caudalmente, em associação com o Ac correspondente. Em 66,6\% no AD e o AE separam-se do Ac em correspondência ao atlas. Em seguida o $\mathrm{Vg}$ passa medialmente às artérias condilar e occipital e seguem no sentido dorsal à artéria carótida comum e ao tronco simpático na região cervical $(96,6 \%)$ (Fig.1 e 2$)$.

\section{Ramificação dos nervos vagos direito e esquerdo}

O Vago divide-se em ramos faríngeos e nervos laríngeos craniais em $86,66 \%$ e $96,6 \%$ dos casos, respectivamente. Os ramos faríngeos originam-se ao nível do atlas $(73,3 \%)$ e da articulação atlantooccipital $(13,3 \%)$ em ambos os antímeros. Os nervos laríngeos craniais, no $\mathrm{AD}$ e no $\mathrm{AE}$, originam-se do tronco dos Vgde (96,6\%) (Fig. 1 e 2).

\section{Trajeto dos ramos retrofaríngeos do nervo vago: ramos farín- geos e nervos laríngeos craniais}

Os ramos faríngeos emitem os ramos faríngeos propriamente ditos e os ramos faringoesofágicos. Em 36,6\% dos casos no $\mathrm{AD}$ e em $35 \%$ no $\mathrm{AE}$, mostram-se localizados medialmente ao tronco simpático (Fig.1 e 2).

Os nervos laríngeo craniais direito e esquerdo, postam-se em $50 \%$ no $\mathrm{AD}$ e $50 \%$ no $\mathrm{AE}$ ventralmente à face lateral da laringe. Estes ramos passam lateralmente ao tronco simpático e medial à artéria carótida comum; acompanham a artéria laríngea cranial lateralmente aos ramos faringoesofágicos alcançando a laringe através da fissura tireóidea, entre os músculos tireofaríngeo e hiofaríngeo (Fig.1 e 2).

\section{Ramificação dos ramos faríngeos dos nervos vago direito e esquerdo: ramos faríngeos propriamente ditos e ramos faringoesofágicos \\ Os ramos faríngeos dão origem aos ramos faringoesofágico direito e esquerdo, seguindo rostralmente como ramos faringoesofágicos direito e esquerdo cujas origens, relacio-}

nam-se ao atlas ou à articulação atlantooccipital em $45 \%$ dos casos no $\mathrm{AD}$ e em $43,3 \%$ no $\mathrm{AE}$. Na mesma porcentagem os ramos faringoesofágicos direito e esquerdo foram encontrados emergindo dos ramos faríngeos (Fig.1 e 2).

\section{Ramificação dos nervos laríngeos craniais}

Uma vez constituídos, os nervos laríngeos craniais dispõem-se ventralmente na parede lateral da faringe, emitem ramos externos e continuam como ramos internos.

\section{Origem individualizada dos ramos dos nervos laríngeos craniais}

Os ramos internos são identificados como a continuação dos nervos laríngeos craniais (100\%) e surgem diretamente do $\mathrm{Vg}$ em $48,3 \%$ dos casos no $\mathrm{AD}$ e no $\mathrm{AE}$. Os ramos externos destacam-se do tronco dos nervos laríngos craniais em 35,5\% no $\mathrm{AD}$ e $36,6 \%$ no $\mathrm{AE}$ e diretamente do $\mathrm{Vg}(8,3 \%$ no $\mathrm{AD}$ e $6,6 \%$ no AE) (Fig.1).

\section{Trajeto das ramificações dos ramos faríngeos direito e esquerdo}

Os ramos faríngeos propriamente dito seguem rostroventralmente cruzando os ramos para o seio carotídeo medialmente e destinam-se aos até os músculos da faringe, palato mole e mucosa da faringe em $25 \%$ dos casos no $\mathrm{AD}$ e $23,3 \%$ no AE. Estes ramos se localizam medialmente aos ramos do seio carotídeo e do NG em ambos os antímeros (20\%) (Fig.1).

Em $40 \%$ dos casos os ramos faringoesofágicos correm na direção ventral dos antímeros esquerdo e direito, cruzam medialmente a artéria carótida comum, rostralmente ao nervo carótido externo, e prosseguem caudalmente em relação à face dorsolateral da faringe. Estes ramos em 30\% das oportunidades, para ambos os antímeros, seguem caudalmente em relação à face dorsolateral da faringe, cruzam lateralmente a artéria faríngea e medialmente a artéria lingual e aos ramos internos e externos dos nervos laríngeos cranias. Situam-se lateralmente dos ramos externos dos nervos laríngeos craniais nos antímeros direito e esquerdo( 20\%) (Fig.1).

\section{Distribuição dos ramos retrofaríngeos e dos ramos faríngeos direito e esquerdo}

Em 25\% dos casos no AD e $23,3 \%$ no AE, os ramos faríngeos propriamente dito seguem em direção dos músculos estilofaríngeo rostral, hiofaríngeo, porção rostral do tireofaríngeo e levantador do véu palatino. Em ambos os antímeros, em $20 \%$ distribuem-se medialmente aos ramos até o seio carotídeo e aos nervos glossofaríngeos (Gf) (Fig.1).

Em 40\% dos casos, nos antímeros direito e esquerdo, os ramos faringoesofágicos, se subdividem em outros ramos nos músculos tireofaríngeo e cricofaríngeo, enquanto em $10 \%$, no $\mathrm{AD}$ e no $\mathrm{AE}$, alcançam os músculos tireofaríngeo, cricofaríngeo e cricotiróideo. Em ambos os antímeros os ramos rostrais dos ramos faringoesofágicos em 76,6\% dos casos atingem os músculos hiofaríngeos (Fig.1).

\section{Trajeto dos nervos laríngeos craniais}

Os ramos externos correm ventralmente em direção à 
faringe. Estes, após suas origens nos nervos laríngeos craniais situam-se na parte lateral do tronco simpático e medialmente à artéria carótida comum, no $\mathrm{AD}(21,6 \%)$ e no $\mathrm{AE}(20 \%)$. Os mesmos ramos, quando se originam da artéria carótida, mostram-se postados medialmente a essa artéria, no $\operatorname{AD}(21,6 \%)$ e no $\mathrm{AE}(23,3 \%)$, terminam nos ramos faringoesofágicos em $20 \%$ dos casos no $\mathrm{AD}$ e $\mathrm{AE}$, ou emitem ramos para os ramos faringoesofágicos e continuam sua trajetória até os músculos tireofaríngeos e cricotireóideo 23,3\% AD e AE (Fig.1).

Os ramos internos dos nervos laríngeos craniais são continuações dos nervos laríngeos craniais.

\section{Distribuição dos ramos dos nervos laríngeos craniais: ra- mos internos e ramos externos}

Observamos em $100 \%$ das amostras que os ramos internos dos nervos laríngeos craniais dividem em ramos rostrais e caudais, cujas ramificações distribuem-se na mucosa laríngea. Em 46,66\% dos casos os ramos externos do nervo laríngeo cranial, em ambos os antímeros, direcionam-se aos faringoesofágicos e aos músculos tireofaríngeo e cricotireóideo. Os ramos externos, direito e esquerdo, abrangem em apenas $40 \%$ os ramos faringoesofágicos direito e esquerdo (Fig.2).

\section{Ramos comunicantes ("anastomoses")}

É grande a ocorrência de anastomoses entre os troncos e as ramificações dos nervos vago e glossofaríngeo que envolve o gânglio cervical cranial na região retrofaríngea. Freqüientemente são observadas entre:

1. Gânglio cervical cranial e o plexo faríngeo (100\%);

2. Ramos externos dos nervos laríngeos craniais direito e esquerdo e ramos faringo-esofágicos dos nervo vago direito esquerdo $(86,6 \%)$;

3. Ramos rostrais dos ramos faringo-esofágicos os plexos faríngeos (bilateralmente: 73,3\%); e,

4. Ramos faringoesofágicos dos nervos vagos e os ramos faríngeos dos nervos glossofaríngeos (bilateralmente 43,3\%).

\section{Resultados histológicos}

A análise histológica dos fragmentos colhidos do $\mathrm{Vg} \mathrm{Gl}$ evidenciou a presença de agregados de corpos de neurônios à esquerda $(3,3 \%)$ e à direita $(1,6 \%)$ entremeados por feixes de fibras nervosas, caracterizando formações ganglionares. As amostras da parede dorsal limítrofe, entre a faringe e o espaço retrofaríngeo, revelaram a existência de glândulas faríngeas (mucosa e serosa), fibras musculares estriadas, tecido adiposo e aglomerados de corpos de neurônios (1,6\% à esquerda) entremeados nas glândulas mistas.

\section{DISCUSSÃO}

Foi observado em nossos resultados que os nervos glossofaríngeo e vago surgem através do forame jugular. Este orificio denominado como "forame jugular" é o mesmo que o adotado por Dougherty et al. (1958), Dyce et al. (1990) e Schwarze \& Schröder (1972) para ruminantes. May (1974) cita que nos ovinos, os Gf e Vg emergem da cavidade craniana pelo 'orifício rasgado posterior' e Chauveau et al. (1905) e Lesbre (1923) pelo 'forame rasgado posterior'.
No trajeto dos Gfde, observou-se que após abandonarem a cavidade craniana, passam em posição lateral ao gânglio cervical cranial, semelhante aos achados de Dougherty et al. (1958) para ovinos, Dyce et al. (1990) para mamíferos domésticos, Godinho (1973a), Godinho et al. (1987) e Getty (1975) para bovinos, ovinos e caprinos. Neste nervo havia uma pequena dilatação, bilateral, em seu tronco, sendo que Simonetta (1932) refere-se para ovinos e bovinos e Ellenberger \& Baum (1932) para bovinos o termo 'gânglio laterofaríngeo'.

Os estudos microscópicos foram realizados nesta região no qual foi observada a presença de aglomerados de corpos celulares de neurônios distribuídos aleatoriamente entre as fibras nervosas. Da mesma forma Banks (1992) cita que os aglomerados de corpos de neurônios fora do sistema nervoso central são chamados de gânglios. Independente do aspecto macroscópico da dilatação, pode existir um gânglio no trajeto do tronco e dos ramos dos nervos aqui estudados fato que coincide com os dados do trabalho de Kozma \& Gellért (1959).

Os Gfde dividem-se em ramos colaterais e ramos terminais, o que assemelha, com as pesquisas de Chauveau et al. (1905), Montané \& Bourdelle (1913, 1917), Dantas et al. (1967), Schwarze \& Schröder (1972) e Zimmerl (1909) enquanto Dobberstein \& Hoffmann (1964) e Ellenberger \& Baum (1932) citam a ocorrência dos ramos lingual e faríngeo.

Os ramos do seio carotídeo são os primeiros a se destacarem do Gf, relato semelhante é apresentado por Godinho et al (1987) e Getty (1975). Quanto aos níveis de origem destes ramos verificaram-se as seguintes descrições: Dyce et al. (1990) em correspondência a junção do tronco do glossofaríngeo aos Vg e acessório; Evans (1993) ao nível do gânglio cervical cranial; Getty (1975) em relação à margem ventral da bolha timpânica para bovinos e caprinos. Dougherty et al. (1958) em ovinos e Godinho (1973a,b) em ruminantes e suínos, descrevem como níveis das origens dos ramos do seio carotídeo a borda ventral da bula timpânica.

Quanto à origem dos ramos faríngeos direito e esquerdo, observou-se que se apresentavam na mesma posição em todas as amostras e emergiam, na maioria dos casos, em correspondência com a face medial do músculo occiptoióideo. Somente Getty (1975), cita que os ramos podem também serem únicos ou duplos com emergência abaixo do ramo do seio carotídeo nos bovinos e únicos com origem próxima e caudalmente ao músculo estilofaríngeo caudal, nos ovinos. Os autores Chauveau et al. (1905), Zimmerl (1909), Lesbre (1923), Bruni \& Zimmerl (1947), Schwarze \& Schröder (1972) e afirmam que os ramos faríngeos emergem em correspondência com a artéria faríngea ascendente, enquanto Dyce et al. (1990) cita como referência, o músculo estiloíoideo.

Os resultados identificaram que os ramos do músculo estilofaríngeo caudal dos Gf destacam-se em correspondência ao terço médio da margem caudal do músculo estilofaríngeo caudal, e com menor freqüência, ao terço distal da margem caudal do músculo estilofaríngeo. Quanto a este ramo apenas May (1974) relatou que o mesmo emerge do $\mathrm{Vg}$ ao nível do osso estiloióideo, enquanto os resultados de Godinho (1973) coincidem com os achados obtidos descritos acima. 
As divisões terminais dos Gfde foram evidenciados em nossos estudo, como: tonsilar, mucosa faríngea e lingual. Godinho et al. (1987) e Getty (1975) fazem menção aos três ramos terminais em ruminantes, como o descrito neste trabalho.

O Vgde emerge da cavidade craniana juntamente com os nervos glossofaríngeo e acessório, como o descrito por Chauveau et al. (1905). O Vg, após separar-se do Ac, passa medialmente da artéria occipital e segue dorsalmente à artéria carótida comum e ao tronco simpático na região cervical. Para Chauveau et al. (1905) o Vg corre no sentido caudal da bolsa gutural e para Dyce et al. (1990) no sentido do gânglio cervical cranial ou gânglio cervical superior (Zimmerl et al. 1909 , Ellenberger \& Baum 1932) e para Schwarze \& Schröder (1972) no sentido dorsal à artéria carótida interna.

Quanto à ramificação dos Vgde, constatou-se a presença de dois ramos retrofaríngeos principais: o ramo faríngeo e $o$ nervo laríngeo cranial. Os resultados deste trabalho coincidem com os dados de autores consultados, à exceção do nervo laríngeo cranial que Chauveau et al. (1905), Lesbre (1923) e Sprague (1944) denominaram nervo laríngeo superior e Bruni \& Zimmerl (1947), May (1974) e Zimmerl (1909) como nervo laríngeo anterior.

Foi observado que os ramos faríngeos originam-se na maioria das vezes, em correspondência ao atlas, e em menor quantidade, ao nível da articulação atlantooccipital. Chauveau et al. (1905), Zimmerl (1909), Lesbre (1923), Bruni \& Zimmerl (1947) e Getty (1975) mencionam que este ramo origina-se em correspondência ao gânglio cervical superior. Os ramos faríngeos seguem de um pequeno tronco, imediatamente após a sua emergência e em correspondência ao Atlas e mostramse localizados em sentido lateral ao tronco simpático; fato evidenciado por Godinho (1973a).

No presente estudo os ramos faríngeos se dividem em dois ramos principais: ramos faríngeos propriamente dito e ramos faringoesofágicos. Zimmerl (1909), Ellenberger \& Baum (1932), Bruni \& Zimmerl (1947) e Schwarze \& Schröder (1972) são unânimes ao se referirem às ramificações dos ramos faríngeos como ramos dorsal e ventral, Getty (1975) considerou como ramos rostral e caudal.

Quanto à origem individualizada dos nervos laríngeos craniais direito e esquerdo, mostramos que na maioria dos casos, destacam-se em correspondência ao atlas, e em menor frequiência, na articulação atlantoaxial. Achados coerentes com os autores: Godinho et al. (1987a), Godinho (1973) e Getty (1975) para ruminantes domésticos.

Os nervos laríngeos craniais, em todas as amostras analisadas, seguem ventralmente na face lateral da laringe. Os ramos caudais ramificam-se na mucosa laríngea, unindo-se às ramificações dos ramos faringoesofágico, e os ramos rostrais emitem diversos ramos que inervam a mucosa laríngea. Estes resultados estão de acordo com os dados citados por Ellenberger \& Baum (1932), Godinho et al. (1987), Getty (1975) e Habel (1970).

Os resultados histológicos realizados do nervo laríngeo cranial revelaram a presença de corpos de neurônios entrelaçados às fibras nervosas que correspondem à origem do nervo.
Informações semelhantes foram descritas, por Dobberstein \& Hoffmann (1964) e Ellenberger \& Baum (1932). Verificou-se que este nervo distribui-se ventralmente na parede lateral da faringe, emitindo os ramos externos que continuam como ramos internos, assemelhando-se aos relatos de Ellenberger \& Baum (1932), Godinho et al. (1987), Getty (1975) e Evans (1993).

De acordo com Ellenberger \& Baum (1932), Schwarze \& Schröder (1972) os ramos faríngeos propriamente dito, descrito em nosso trabalho é designado como ramo dorsal, e distribui-se nos músculos consctrictores faríngeos e na mucosa faríngea.

Outro aspecto merecedor de comentário é a grande ocorrência de anastomoses entre os troncos e as ramificações dos nervos glossofaríngeo e vago, na, região retrofaríngea envolvendo também o gânglio cervical cranial, não sendo descrito pelos tratadistas consultados. Sugere-se que a presença destas anastomoses se traduz em complexos aspectos morfo-funcionais, que somente poderiam ser melhores especificados por outros métodos de estudo.

Os dados apresentados neste estudo contribuirão em procedimentos cirúrgicos e anestésicos, relacionados como os vago e nervo glossofaríngeo na região retrofaríngea, preservando de tal modo as estruturas, evitando-se, assim, complicações pós-operatórias atribuídas às lesões destes nervos. Torna-se recomendável a pesquisa sistemática sobre tais aspectos, não apenas em ovinos, mas também em outras espécies animais assim como sua inserção no conteúdo das disciplinas básicas dos cursos de medicina veterinária.

\section{CONCLUSÃO}

Concluiu-se que não existem diferenças marcantes no comportamento dos nervos glossofaríngeos e vago na região retrofaríngea entre as espécies consideradas de ovinos da raça Santa Inês, e esta informação é importante como subsídio para abordagens clínicas ou cirúrgicas que envolvam as estruturas estudadas.

\section{REFERÊNCIAS}

Banks W.J. 1992. Sistema nervoso, p.324 In: Banks W.J. (ed.), Histologia Veterinária Aplicada. Manole, São Paulo.

Bruni A.C. \& Zimmerl U. 1947. Nervi encefalici, p.581-587. In: Bruni A.C. \& Zimmerl U. (ed.), Anatomia degli Animali Domestici. Vol.2. Casa Editrici Dottor Francesco Vallardi, Milano.

Chauveau A., Arloing S. \& Lesbre F.X. 1905. Traité d'Anatomie Comparée des Animaux Domestiques. Vol.2. 5ํㅜㄹ ed. J.B. Bailliére, Paris, p.524-530.

Dantas A.M., Benevento R. \& Neto L. 1967. Estudo funcional e anátomoclínico do nervo glossofaríngico. Revta Bras. Oftalmol. 26(4):69-76.

Davidson H.P., Rebhun W.C. \& Habel R.E. 1981. Pharyngeal trauma in cattle. Cornell Vet. 71:15-25.

Dobberstein J. \& Hoffmann G. 1964. Lehrbuch der Vergleichenden Anatomie der Haustiere. Vol.3. S.Hirzel, Leipzig, p.163-165.

Dougherty R.W., Habel R.E. \& Bond H.E. 1958. Esophageal innervation and the eructation reflex in sheep. Am. J. Vet. Res. 40:118-121.

Dyce K.M., Sack W.O. \& Wensing C.J.G. 1990. Tratado de Anatomia Veterinária. Guanabara Koogan, Rio de Janeiro, p.41-42, 216.

Ellenberger W. \& Baum H. 1932. Handbuch der Vergleichenden Anatomie der Haustiere. Springer Verlag, Berlin, p.951-953. 
Evans H.E. 1993. Miller's Anatomy of the Dog. $3^{\text {rd }}$ ed. W.B. Saunders, Philadelphia, p.980, 982.

Getty R. 1975. Generalidades sobre o coração e os vasos sangüíneos, p.160. In: Sisson/Grossman Anatomia dos Animais Domésticos. Vol.1. 5 $5^{\underline{a}}$ ed. Guanabara Koogan, Rio de Janeiro, p.160.

Godinho H.P. 1973a. The glossopharyngeal and vagus nerves in the retropharyngeal region of goat, sheep and cattle. Anat. Hist. Embryol.. 2:120-126.

Godinho H.P. 1973b. Nerves of the retropharyngeal region in swine. Arqs Esc. Vet. Univ. Fed. Minas Gerais 25(1):49-53.

Godinho H.P., Cardoso F.M. \& Nascimento J.F. 1987. Anatomia dos Ruminantes Domésticos. Universidade Federal de Minas Gerais, Belo Horizonte, p.206.

Habel R.E. 1970. Guide to the Dissection of Domestic Ruminants. $2^{\text {nd }}$ ed. Ithaca, New York, p.119, 128-130

Kozma M. \& Gellért A. 1959. Vergleichende histologische Untersuchungen über die mikroskopischen Ganglien und Nervenzellen des Nervus glossopharyngeus. Ann. Anat. 106:38-49.

Lesbre F.X. 1923. Précis d'Anatomie Comparée des Animaux Domestiques. Vol.2. J.B. Baillière, Paris, p.32-639.
May N.D.S. 1974. Anatomia del Ovino. Hemisferio Sur, Buenos Aires, p.301403.

Montané L. \& Bourdelle E. 1913. Anatomie Régionale dês Animaux Domestique. Vol.1. J.B. Baillièr, Paris, p.472-475.

Montané L. \& Bourdelle E. 1917. Anatomie Régionale dês Animaux Domestique. Vol.2. J.B. Baillière, Paris, p.432-433.

Moore K.L. 1990. Anatomia Orientada para a Clínica. $2^{\underline{a}}$ ed.Guanabara Koogan, Rio de Janeiro, p.717-719.

Simonetta B. 1932. Origine e Sviluppo del Nervo Terminale nei Mammiferi; sua Funzione e suoi Rapporti con l'Organo dijacobson. Anat. Embryol. 97(3-4):425-463.

Schwarze E. \& Schröder L. 1972. Compendio de Anatomia Veterinária. Vol.4. Acribia, Zaragoza, p.103-107.

Sprague J.M. 1944. The innervation of the pharynx in the rhesus monkey, and the formation of the pharyngeal plexus in primates. Anat. Rec. 90:197207.

Stocker S., Geissbühler U. \& Steiner A. 1997. Retropharyngealer Abszess beim Rind: ein Fallbericht. Schweiz. Arch. Tierheilkd. 139:314-318.

Zimmerl U. 1909. Trattato di Anatomia Veterinária. Vol.3. Francesco Vallardi, Milano, p.202-210. 